\title{
Two-Dimensional LMS Adaptive Linear Phase Filters
}

\author{
Soo-Chang Pei, Ching-Yung Lin and Chien-Cheng Tseng \\ Department of Electrical Engineering, National Taiwan University, \\ Taipei, Taiwan, R.O.C.
}

\begin{abstract}
The two-dimensional least mean square (2D LMS) adaptive filters have been recently used in the image processing applications for reducing the noise. In this paper, a new two-dimensional LM$S$ algorithm is proposed. For the special desires to the linear phase constraint during filtering the images, an additional linear phase constraint is added to the existing 2D LMS algorithms. Compare with the conventional algorithms, the results show that the proposed algorithm is much more efficient both in computation time and memory storage.
\end{abstract}

\section{Introduction}

FIR filters with linear phase response are of great importance in designing filters [1] and in other signal processing systems where frequency dispersion, due to nonlinear phase characteristic in passband, is undesirable. Applications' examples are in the areas such as system identification [2], communication [3] and high resolution harmonic analysis [2]. In image processing, the linear phase characteristic appears to be more critical than other applications [4]. Our visual world consists of lines, scratches, etc. A nonlinear phase disperses different frequency components that make up the lines and scratches, as a result, this will bring seriously blur to the image. Thus, letting a image processed by a linear phase filter is inherently necessary. 0.7803-1254-6/93\$03.00 1993 IEEE
Recently, 2D adaptive algorithm based on the method of steepest descent has been develope$\mathrm{d}$ and applied to the noise reduction in images [5][6][7]. These adaptive algorithms enable the filter to have better tracking performance in nonstationary images than the conventional Wiener Filter which is designed under the assumption of stationary signal and noise. Although so much good adaptive algorithms have been develpoed, the filters are all with nonlinear phase response. In order to improve the performance of algorithm$s$, the linear phase constraint must be added. This is the purpose for this paper.

It is well-known that the linear phase FIR filters have symmetric (half- symmetric) impulse response. This characteristic is quite useful in simplifing design and implementation of a filter. Thus, 2D LMS linear phase filter will only need a half of the computation and memory of nonlinear phase 2D LMS filter. Moreover, when 2D linear phase LMS algorithm is applied to the noise reduction in image, its mean square error (MSE) is smaller than that of the current 2D LMS algorithm, due to the linear phase constraint is important in filtering an image.

\section{2D LMS linear phase filter}

The 2D FIR digital filters are generally represented by the following convolution form : 


$$
y(m, n)=\Sigma_{k=0}^{N-1} \Sigma_{l=0}^{N-1} h(k, l) x(m-k, n-l)
$$

where the size of the input signal $x(m, n)$ is assumed to be $M \times M$ and the size of the impulse response $h(k, l)$ of the filter is assumed to be $N \times$ $\mathrm{N}$. For convenience, we will only consider $\mathrm{N}$ is odd number case. When FIR filter is constrained to be linear phase, it must satisfy the following half-symmetric condition (Fig.1) :

$$
h(k, l)=h(N-1-k, N-1-l) \quad,(k, l) \in T
$$

where

$$
\begin{aligned}
T= & \left\{0 \leq k \leq \frac{N-1}{2}, 0 \leq l \leq \frac{N-1}{2}\right\} \cup \\
& \left\{\frac{N+1}{2} \leq k \leq N-1,0 \leq l \leq \frac{N-3}{2}\right\}
\end{aligned}
$$

In order that the filter output $y(m, n)$ can track the desired input $d(m, n)$, we proposed the following 2D LMS linear phase algorithm :

$$
\begin{aligned}
& h_{j+1}(k, l)= h_{j}(k, l)+\nu e(m, n) \times \\
&\{x(m-k, n-l)+x(m+k-N+1, n+l-N+1)\} \\
& \quad \text { initial value : } h_{0}(k, l)=\frac{1}{N^{2}}
\end{aligned}
$$

where

$$
\begin{gathered}
\mathrm{j}: \text { iteration number } \\
\nu: \text { step size } \\
e(m, n)=d(m, n)-y(m, n)
\end{gathered}
$$

The iteration number used in [5] is $j=m \times M+n$ (Fig.2-(a)). This processing path updates the filter taps line by line. Thus, the filter taps used on the first pixel of a line are just right the ones adapted by the last pixel of the adjacent fron$\mathrm{t}$ line. Since 2D images are not continuous in this one-dimensional update path, it will cause a sudden jump to the adaption of the filter taps. The algorithm proposed by Ohlii suggested a two-dimensional update path [6] (Fig.2-(b)), but it is not very efficient. From this speed consideration, we can still using one-dimensional update path but with the iteration number $\mathrm{j}$ as follows (Fig.2-(c)):

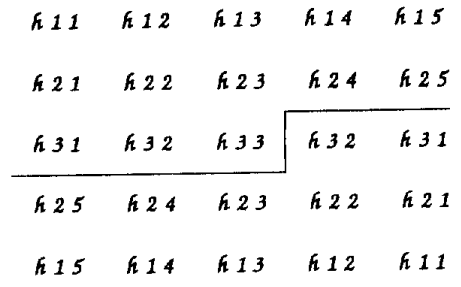

Fig.1 A $5 \times 5$ half-symmetric filter taps.

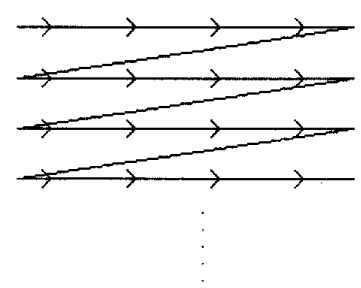

(a) Hadhoud's

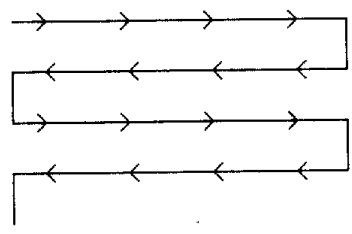

(c) Proposed

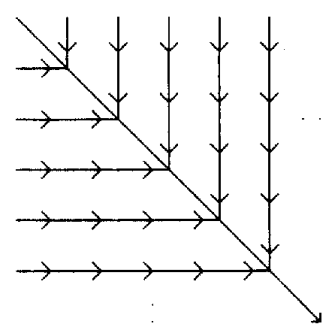

(b) Ohki's

$$
\begin{array}{rr}
j=m \times M+n, & \mathrm{~m} \text { is even. } \\
m \times M+(M-n), & \mathrm{m} \text { is odd. }
\end{array}
$$

That means we update the filter taps with the path from left to right if $m$ is even and from right to left if $\mathrm{m}$ is odd.

During the adaptive process, the following constraint has to be added :

$$
\sum_{k=0}^{N-1} \Sigma_{l=0}^{N-1} h_{j}(k, l)=1
$$

This normalization procedure preserves the image local mean value, which also has been used in nonlinear phase 2D LMS algorithm [5].

\section{2D adaptive linear phase enhancer}

In order to show the performance of the proposed algorithm, we compare it to the existing two algorithms : 


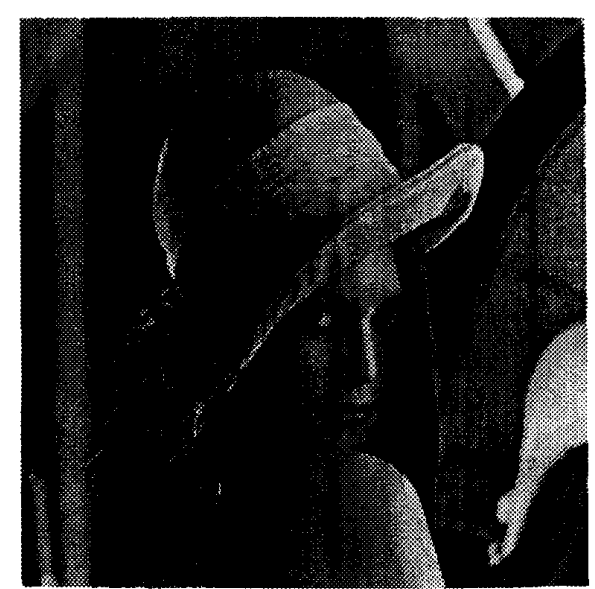

Fig. 3 The source image "Lena"

\begin{tabular}{|c|c|c|}
\hline & Multiplication & $\begin{array}{c}\text { Memory } \\
\text { (maximum) }\end{array}$ \\
\hline ladhoud's TDLMS & $N^{2} \times 2$ & $N^{2}$ \\
\hline Ohki's Algorithm & $N^{2} \times 4$ & $N^{2} \times(2 M-3)$ \\
\hline roposed Algorithm & $\frac{N^{2}+1}{2} \times 2$ & $\frac{v^{2}+1}{2}$ \\
\hline
\end{tabular}

The saves in both multiplications and menories can be observed. For example, if a $256 \times 256$ contaminated image is processed by a $3 \times 3$ filter, the CPU time ratio of Hadhoud's, Ohlij's and proposed algorithm is $1.8: 3.6: 1$ and the need of storage memory is $1.8: 101.8: 1$. It can be shown that the proposed algorithm is much more efficient in computation time and memory storage. Since the need of large memory will cause large data transfer time between $\mathrm{CPU}$ and the storage elements. This linear phase property brings us more benefits while excuting the algorithm in a machine with limited speed and memory such as PC.

An example is shown in Fig.3 - Fig.8. and the parameters used are :

"Lena" image size : $256 \times 256$ (Fig.3).

Filter size : $3 \times 3$.

The image is degraded to $\mathrm{SNR}=12.57 \mathrm{~dB}$ (Fig.4).

The best results of the three filters individually are as follows: Fig. 5 is the image processed by the Hodhoud's algorithm with step size $\nu=7 \times 10^{-10}$ and gets $\mathrm{SNR}=13.94$. The Ohli's one with step

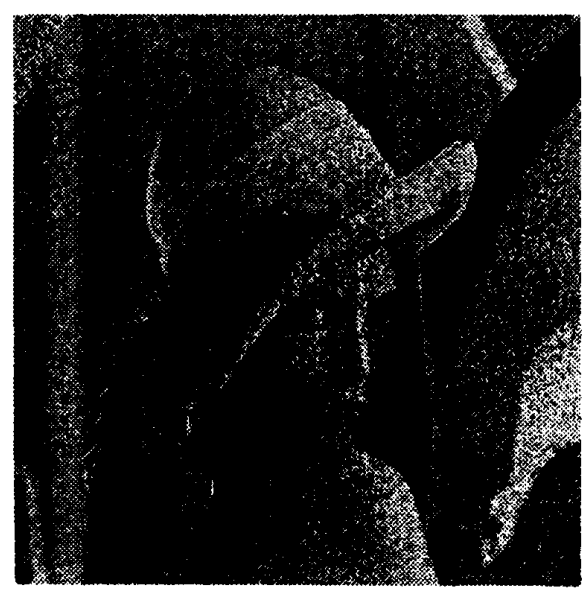

Fig. 4 The degraded image with $\mathrm{SNR}=12.57 \mathrm{~dB}$

size $\nu=5 \times 10^{-7}$ and SNR=14.20 is on Fig.6. Finally, Fig. 7 shows the one processed by the proposed algorithm with step size $\nu=7 \times 10^{-9}$ and gets $\mathrm{SNR}=13.56$. A comparision of SNR to different step sizes is on Fig.8.

Although the SNR improvement the proposed one achieves is less than the others, but the simulation pictures show that the quality of the proposed algorithm is similar to the Ohki's and Hadhoud's by visual observation. However, it is much faster than the others.

\section{Conclusion}

The motivation of adding the linear phase constraint to the Two-dimensional LMS adaptive filter has brought some benefits to us, especially in the computation time and memory storage. Although the image improvement shown with this additive constraint is not so evident, however, this proposed algorithm is very attractive for its speed and efficiency.

\section{References}

[1] Alan V. Oppenheim and Ronald W. Schafer. "Discrete-Time Signal Processing." Englewood Cliffs, NJ: Prentice-Hall 1989.

[2] S. L. Marple, "Fast Algorithm for Linear Prediction and System Identification Filters 


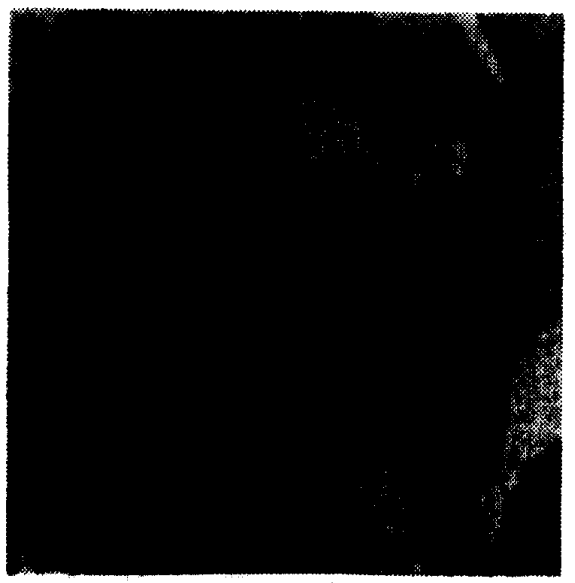

Fig.5 The image processed by the Hadhoud's algorithm with step size $\nu=7 \times 10^{-10}, \mathrm{SNR}=13.94$.

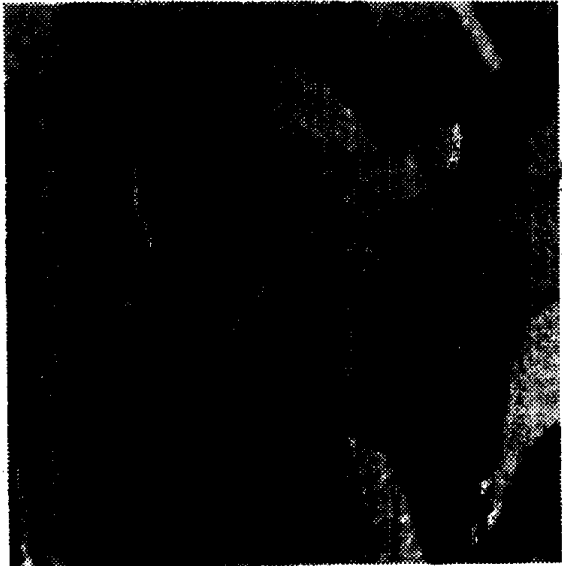

Fig. 6 The image processed by the Ohki's algorith$\mathrm{m}$ with step size $\nu=5 \times 10^{-7}, \mathrm{SNR}=14.20$.

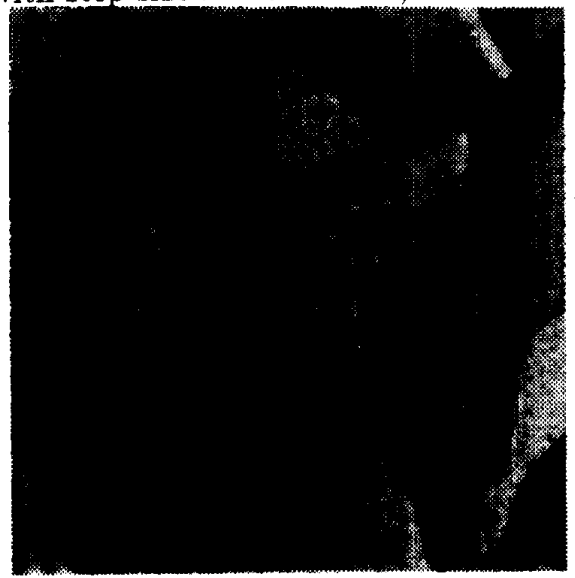

Fig.7 The image processed by the proposed algorithm with step size $\nu=7 \times 10^{-9}, \mathrm{SNR}=13.56$.

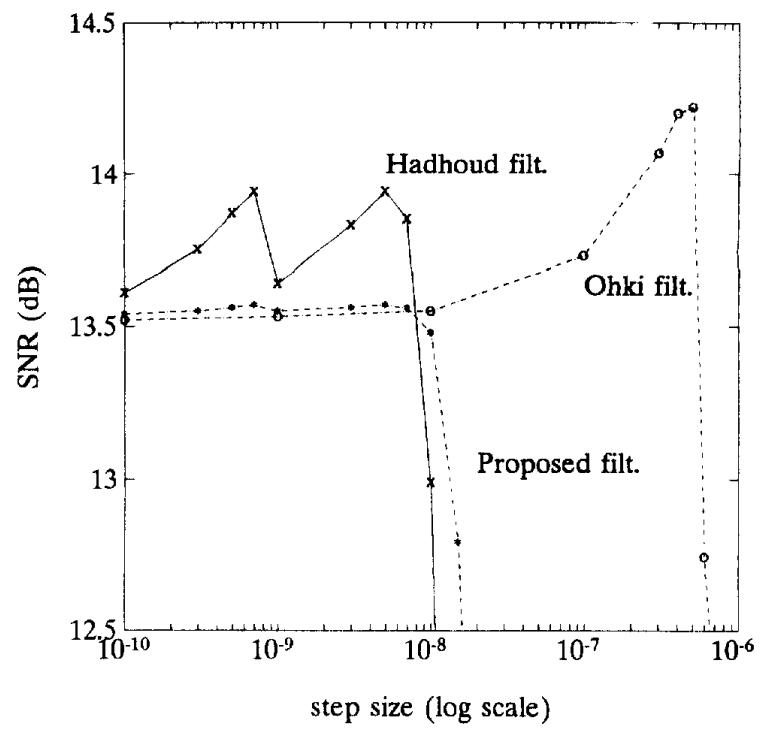

Fig. 8 The comparisons of the three algorithms

with Linear Phase," IEEE Trans. on Acoustic, Speech, Signal Processing, vol. ASSP-30, No. 6, pp. 942-953, December 1982.

[3] K. Berberidis and S. Theodoridis, "New Levinson, Shur and Lattice Type Algorithms for Linear Phase Filtering," IEEE Trans. on Acoustic, Speech, Signal Processing, vol. ASSP-38, No. 11, pp. 1879-1892, November 1990.

[4] J. S. Lim, "Two-Dimensional Signal and Image Processing," Englewood Cliffs, NJ: PrenticeHall 1990.

[5] Monhiy M. Hadhoud and David W. Thomas, "The Two-Dimensional Adaptive LMS (TDLMS) Algorithm," IEEE Trans, on Circuit and Systems, vol. 35, No. 5, May 1988.

[6] Makoto Ohki and Sumihisa Hashiguchi, "TwoDimensional LMS Adaptive Filters," IEEE Trans. on Consumer Electronics, vol. 37, No. 1, February 1991.

[7] Wasfy B. Michael and Shomit M. Ghosh, "Two-Dimensional Variable Step-Size Sequential Adaptive Gradient Algorithms with Applications," IEEE Trans. on Circuit and Systems, vol. 38, No. 12, December 1991. 